\title{
The novel Jab1 inhibitor CSN5i-3 suppresses cell proliferation and induces apoptosis in human breast cancer cells
}

\author{
H. XIAO ${ }^{1}$, F. X. CLARET ${ }^{2}$, Q. SHEN 3 ,*
}

${ }^{1}$ Department of Gastroduodenal and Pancreatic Surgery, Hunan Cancer Hospital and the Affiliated Cancer Hospital of Xiangya School of Medicine, Central South University, Changsha, Hunan Province 410013, China; ${ }^{2}$ Department of Systems Biology, The University of Texas MD Anderson Cancer Center, Houston, TX 77030, USA; ${ }^{3}$ Department of Oncology, Tongji Hospital, Tongji Medical College, Huazhong University of Science and Technology, Wuhan 430030, China

*Correspondence: Shenqian@hust.edu.cn

Received October 16, 2018 / Accepted January 8, 2019

\begin{abstract}
Jab1 is a vital subunit of the CSN family and is reported to be overexpressed in numerous cancer types. Due to the importance of Jab1/CSN5 in cancer cell proliferation and survival, Jab1 is considered a promising therapeutic target. Therefore, we evaluated the anticancer effect of the novel Jab1 inhibitor CSN5i-3 in breast cancer cells. In our study, we found that Jab1 was overexpressed in breast cancer tissues and was correlated with poor prognosis in human breast cancer patients. An MTS assay revealed that CSN5i-3 suppressed cell proliferation in the breast cancer cell lines BT474 and SKBR3. We also found that CSN5i-3 significantly induced apoptosis and G1 phase cell cycle arrest in breast cancer cells. A mechanistic investigation revealed that CSN5i-3 inhibited Jab1 expression and increased the level of apoptosis marker cleaved PARP and the cell-cycle-related protein p27 in BT474 and SKBR3 cells. A nude mouse xenograft model also indicated that CSN5i-3 exerted a potent anticancer effect in vivo. Overall, our study suggests that the Jab1 inhibitor CSN5i-3 might be a promising agent for the breast cancer treatment in humans and should be further studied.
\end{abstract}

Key words: Jab1, CSN5i-3, Breast cancer

Breast cancer is the most commonly diagnosed cancer in women and the second leading cause of cancer-related death in women worldwide $[1,2]$. HER2-positive breast cancer is characterized by the amplification or overexpression of the HER2 oncogene/protein and accounts for approximately $15-20 \%$ of all breast cancer cases $[3,4]$. Trastuzumab is the first targeted therapy drug that has been approved for HER2-positive breast cancer [5]. Though trastuzumab significantly improves patients' overall survival, resistance to the drug is inevitable [6]. Thus, it is necessary to develop novel promising therapeutic drugs for breast cancer.

c-Jun activation domain-binding protein-1 (Jab1), encoded by the COPS5 gene, was initially identified as a coactivator of c-Jun [7], a transcription factor that is involved in numerous cellular processes $[8,9]$, such as cell proliferation and tumorigenesis [10-12]. Later, Jab1 was discovered to be the fifth member and integral component of the photomorphogenic-9 (COP9) signalosome (CSN) complex [13]. CSN is a multifunctional protein complex that is involved in modulating signal transduction, gene transcription, and protein stability. For this reason, Jab1 is also referred to as CSN5. Jab1 contains 3 distinct domains, namely, the c-Jun binding domain (JBD), the Mpr1-Pad1-N-terminal (MPN) domain and the C-terminal domain [9]. The MPN domain contains the metalloenzyme motif (JAMM), which is essential for mediating the interaction between Jab1/CSN5 and other proteins and for its isopeptidase activity $[14,15]$. In cancer, Jab1 has been recognized as a multifunctional protein that plays vital roles in DNA damage response, oncoimmunity and other biological processes [16-18].

Because of the importance of Jab1, a specific Jab1 inhibitor (CSN5i-3) has been designed [19], and it is also reasonable to evaluate the therapeutic potential of this inhibitor in cancer cells. In our study, we detected the anticancer effect of CSN5i-3 in breast cancer cell lines and found that CSN5i-3 inhibited the proliferation and significantly induced apoptosis in vitro and in vivo. Significant G1 phase arrest was also observed in CSN5i-3-treated cells. On the basis of our data, we proposed that the Jab1 inhibitor CSN5i-3 might be a valuable therapeutic agent in human breast cancer. 


\section{Materials and methods}

Cell culture. The BT474 and SKBR3 human breast cancer cell lines were obtained from ATCC. BT474 and SKBR3 cells were cultured in Roswell Park Memorial Institute 1640 medium (RPMI 1640) with 10\% fetal bovine serum (FBS), penicillin $(100 \mathrm{U} / \mathrm{ml})$, and streptomycin $(100 \mu \mathrm{g} / \mathrm{ml})$. All the cells were incubated at $37^{\circ} \mathrm{C}$ in a humidified chamber supplemented with $5 \% \mathrm{CO}_{2}$.

Cell proliferation MTS assay. Cell viability was assessed using a 3-(4,5-dimethylthiazol-2-yl)-5-(3carboxymethoxyphenyl)-2(4-sulfophenyl)-2H-tetrazolium (MTS) assay (Promega, USA). Cells were seeded 24 hours before the indicated treatments. The cells were seeded onto 96-well cell-culture plates overnight before CSN5i-3 treatment. Cell proliferation was accessed by adding 20 MTS solution into each well. The color that developed was read with an enzyme-linked immunosorbent assay reader at $490 \mathrm{~nm}$. The results are expressed as the mean values \pm standard deviations.

Apoptosis assay. To assess apoptosis effects, BT474 and SKBR3 cells were seeded in a 6-well plate. After exposure to CSN5i-3 for 24 hours, cells were stained with Annexin V-FITC and analyzed by FACSCalibur (Becton Dickinson) to assess the apoptotic cell percentages.

Cell cycle arrest. For cell cycle arrest analysis, BT474 and SKBR3 cells were seeded in a 6-well plate and treated with CSN5i-3 for 24 hours. Then, cells were removed and fixed with $70 \%$ ethanol overnight at $4{ }^{\circ} \mathrm{C}$. The cells were washed with PBS, resuspended in $400 \mu \mathrm{l}$ of PI/RNase staining buffer
(Becton-Dickinson), and incubated in the dark for $30 \mathrm{~min}$. Then, the cells were counted on a FACSCalibur (Becton Dickinson). The results were analyzed using Cell Quest (Becton Dickinson) software.

Western blotting assay. The quantified cell lysates were separated by SDS-PAGE, transferred to a PVDF membrane (GE Healthcare) and blocked with 5\% nonfat milk in PBS with $0.1 \%$ Tween-20 (PBST). The membranes were incubated with primary antibodies overnight at $4{ }^{\circ} \mathrm{C}$ and then washed with PBST. The membrane was incubated with secondary antibody for 1-2 hour(s) at room temperature. Immunoreactive bands were detected with horseradish peroxidase-conjugated secondary antibodies using the Western Lightning Chemiluminescence Reagent (Thermo Scientific).

Xenograft assay. Female $\mathrm{BALB} / \mathrm{c}$ nude mice that were 6-7-weeks old and weighed 15.2-17.3 g were obtained from HFK Biotechnology (Beijing). BT474 cells $\left(1 \times 10^{6}\right.$ cells per mouse, in $100 \mu \mathrm{l}$ of PBS) were subcutaneously injected to the mammary fat pad area of each mouse. When the tumor size reached an average volume of $100 \mathrm{~mm}^{3}$, the mice were randomly divided into two groups (each group consisted of 6 mice). Group A was treated with CSN5i-3 $100 \mathrm{mg} / \mathrm{kg} / \mathrm{d}$; Group B was treated with saline as a negative control. The animals' body weights and tumor volumes were recorded every three days.

Bioinformatic analysis. The TCGA mRNA sequence data [16] (www.cbioportal.com) were applied to estimate the relative expression level and prognostic significance of JAB1 in breast cancer tissue compared with that in normal tissue.
A

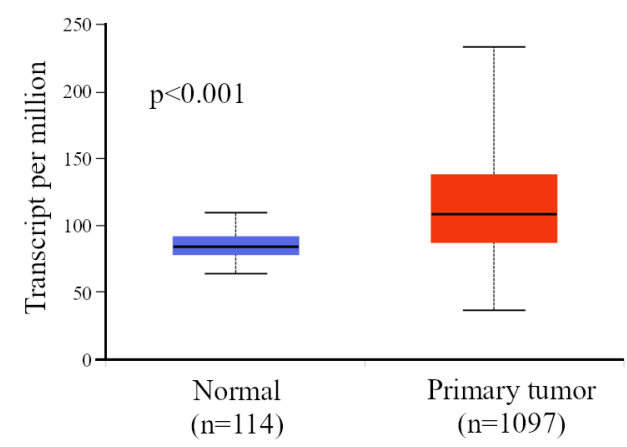

C

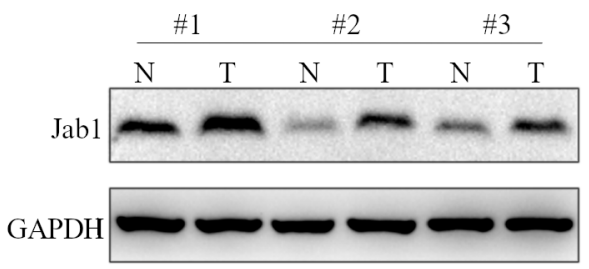

B

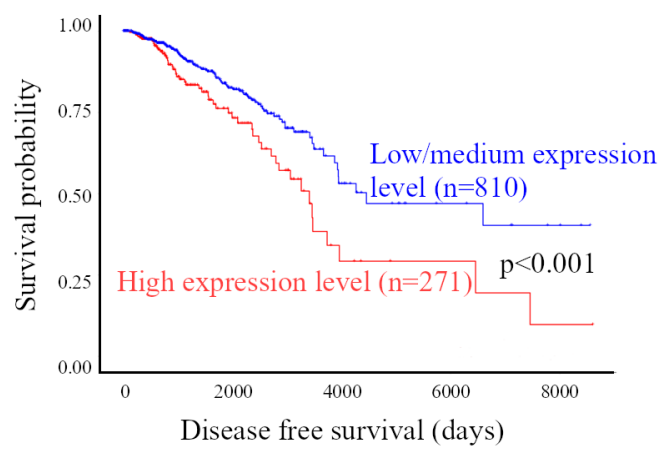

Figure 1. The expression of Jab1 and its correlation with clinical characteristics of breast cancer. A) Compared to its expression in 114 normal tissue samples, the expression of Jab1 was higher in 1097 breast cancer tissue samples $(p<0.01$, cut off value=1.5). B) The DFS rates of patients with higher Jab1 expression were shorter than those of the patients with lower Jab1 expression $(\mathrm{p}<0.01)$. C) Western blot showed that the expression of Jab1 in breast cancer tissues was higher than that in normal tissues from three breast cancer patients. 
Statistical analysis. Data are presented as the mean \pm standard deviation (SD). Data were analyzed by one-way ANOVA followed by the Scheffe and Tukey tests using SPSS 19.0 software. Significance was indicated by $\mathrm{p}<0.05$.

\section{Results}

Jabl is overexpressed and correlated with poor prognosis in breast cancer. To investigate the expression of Jab1 and its potential role in breast cancer, we analyzed the TCGA mRNA sequence data and found that Jab1 was significantly overexpressed in breast cancer tissue $(\mathrm{p}<0.001)$ (Figure $1 \mathrm{~A})$,

BT474

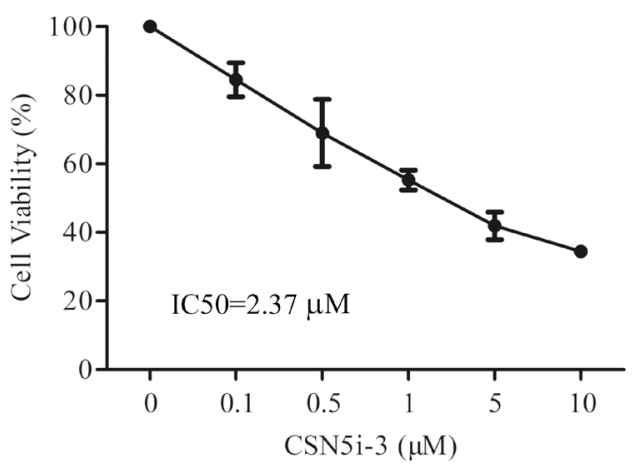

and the disease-free survival rate (DFS) of the patients with higher levels of Jab1 expression were shorter than that of patients with lower Jab1 levels $(\mathrm{p}<0.05)$ (Figure 1B). These data indicated that Jab1 might be a potential therapeutic candidate in breast cancer.

The Jab1 inhibitor CSN5i-3 suppresses the proliferation of human breast cancer cells. To detect the anticancer effect of CSN5i-3 in breast cancer cells, the BT474 and SKBR3 human breast cancer cell lines were treated with the indicated concentrations of CSN5i-3 for 3 days. An MTS assay was then performed to detect the effect of CSN5i-3 on cell proliferation. As shown in Figure 2 CSN5i-3 treatment

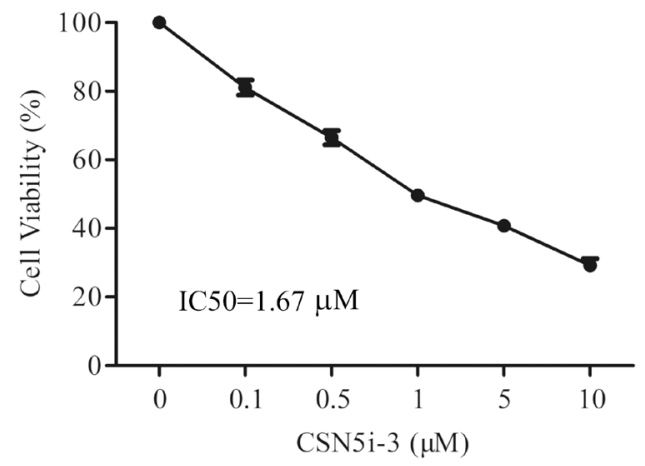

Figure 2. The effect of CSN5i-3 on cell proliferation in human breast cancer cells. After treatment with the indicated concentrations of CSN5i-3 for 3 days, the proliferation of BT474 and SKBR3 cells was significantly suppressed in a dose-dependent manner, and the $\mathrm{IC}_{50}$ was $2.37 \pm 1.15 \mu \mathrm{M}$ in $\mathrm{BT} 474$ cells and $1.67 \pm 1.11 \mu \mathrm{M}$ in SKBR3 cells.
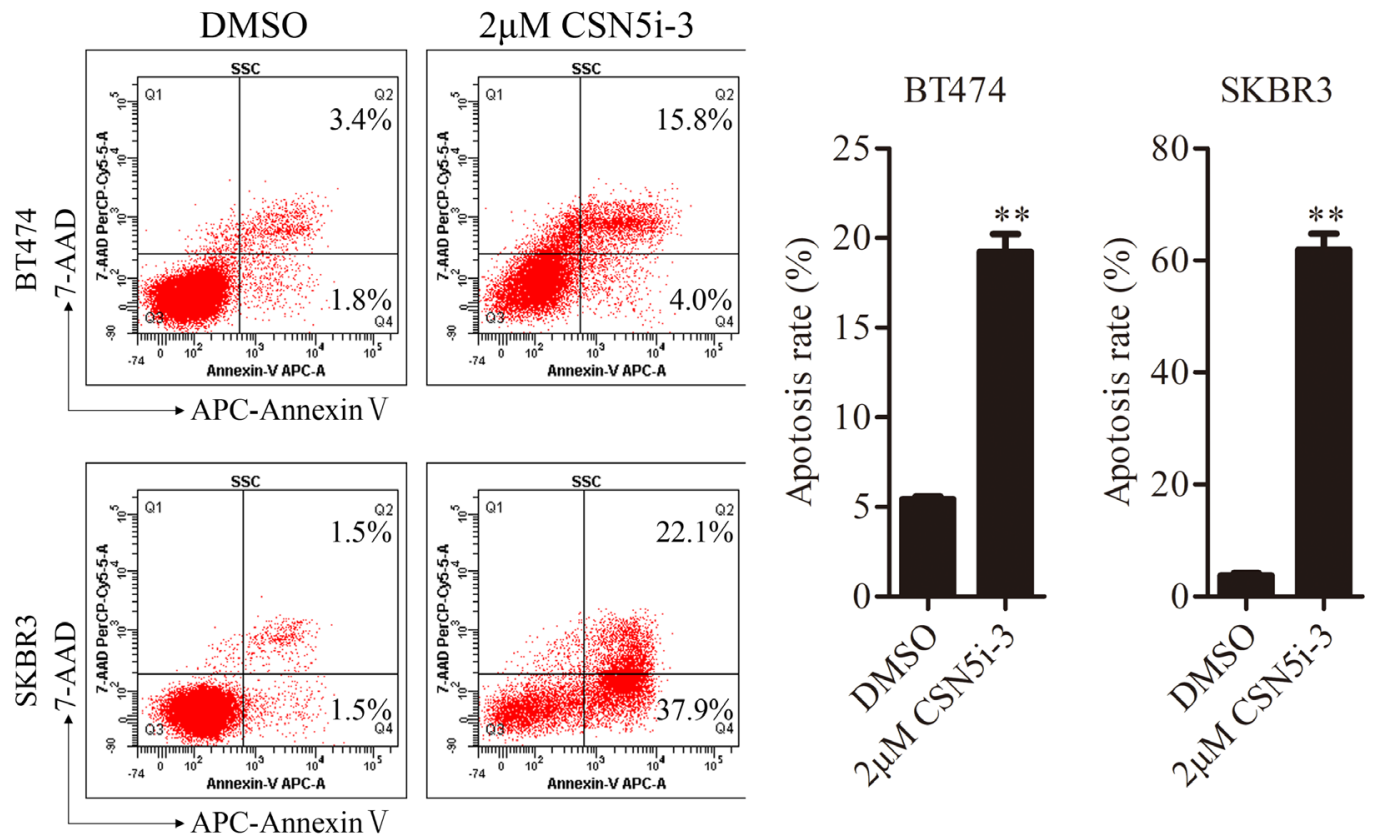

Figure 3. The apoptosis induction effect of CSN5i-3 in human breast cancer cells. A) BT474 and SKBR3 cells were treated with $2 \mu \mathrm{M}$ of CSN5i-3 for 24 hours, and Annexin V/PI staining was performed to detect the apoptosis induction effect using flow cytometry. B) In the BT474 cells, the apoptosis rate was $19.23 \pm 0.9939 \%$ in the treated group $(p<0.01$ ), and in the SKBR3 cells, the apoptosis rate was $61.90 \pm 2.848 \%$ in treated group ( $<<0.01$ ), which were higher than the values observed in the control groups ${ }^{* *}$ indicates that the $\mathbf{p}$-value was less than 0.001$)$. 

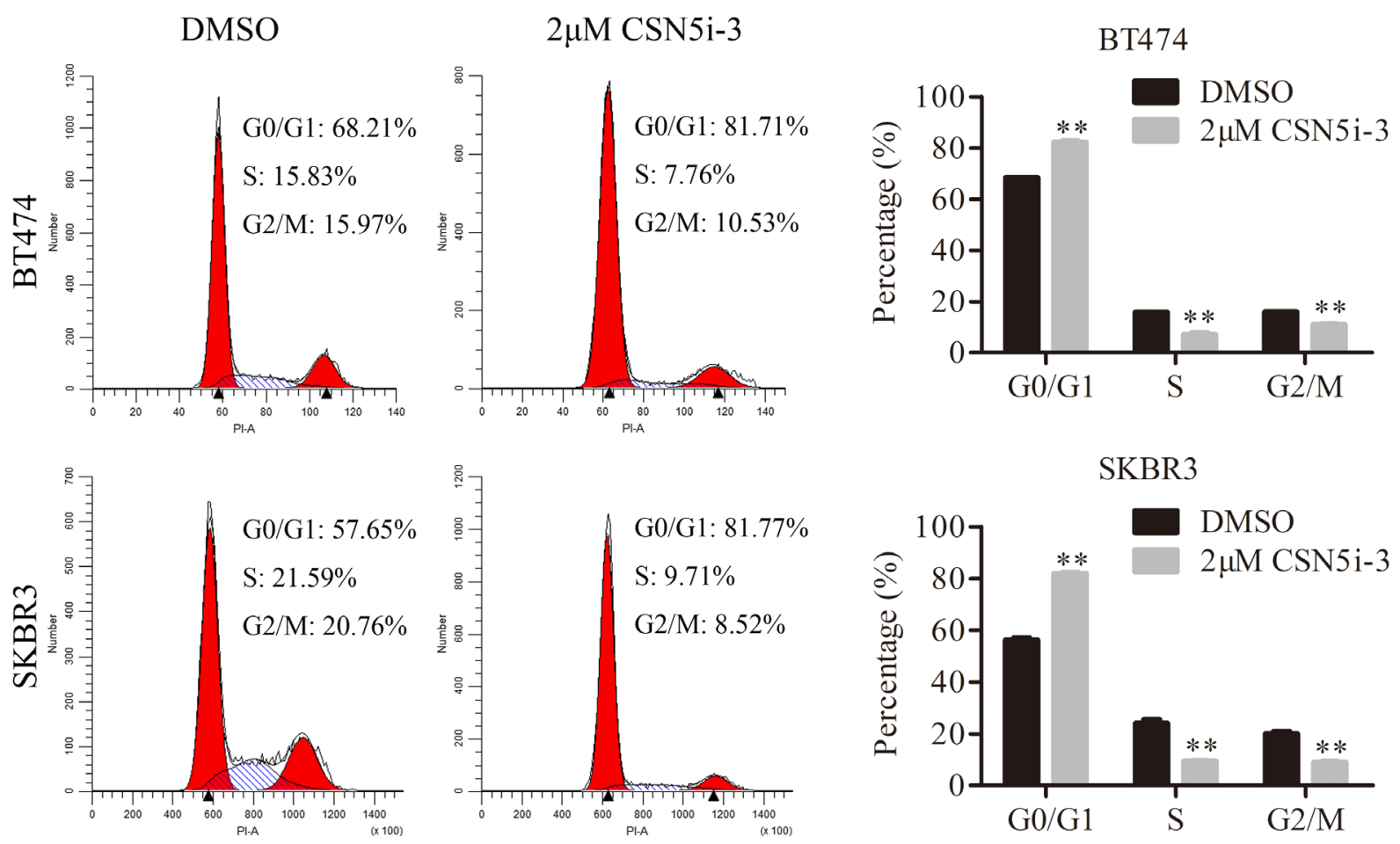

Figure 4. The cell cycle distribution effect of CSN5i-3 in human breast cancer cells. A) PI staining indicated that the $2 \mu \mathrm{M}$ CSN5i-3 exposure resulted in a significant G1 phase arrest in both BT474 and SKBR3 cells. B) After treatment with CSN5i-3 for 24 hours, the ratio of cells in the G1 phase was significantly higher than that in the control groups in the BT474 $(82.22 \pm 0.51 \%$ vs $68.31 \pm 0.095 \%, p<0.01)$ and SKBR3 $(81.82 \pm 0.4452 \%$ vs $56.13 \pm 0.7632 \%$, $\mathbf{p}<0.01)$ cells $\left({ }^{* *}\right.$ indicates that the $\mathbf{p}$-value was less than 0.001$)$.

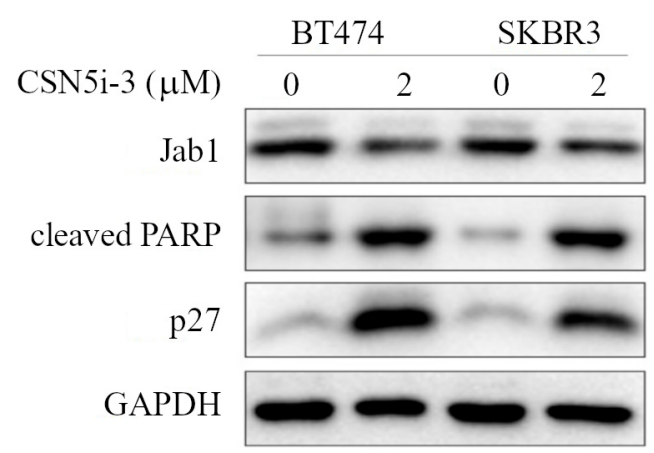

Figure 5. The effect of CSN5i-3 treatment on the expression of Jab1, p27 and cleaved PARP. After treated with CSN5i-3 for 24 hours, the expression of Jab1 was reduced and the protein levels of the cell-cycle-related protein p27 and the apoptosis marker cleaved PARP were significantly increased in the BT474 and SKBR3 cells.

suppressed the proliferation of breast cancer cells in a dosedependent manner, and the $\mathrm{IC}_{50}$ was $2.37 \pm 1.15 \mu \mathrm{M}$ in BT474 cells and $1.67 \pm 1.11 \mu \mathrm{M}$ in SKBR3 cells. Based on our data, targeting Jab1 can significantly inhibit the proliferation of breast cancer cells.

The Jab1 inhibitor CSN5i-3 significantly induces cell apoptosis in human breast cancer cells. Then, we tested the apoptosis induction effect of CSN5i-3 in breast cancer cells.
We treated BT474 and SKBR3 cells with $2 \mu \mathrm{M}$ CSN5i-3 for 24 hours and found that Jab1 inhibition led to significantly enhanced apoptosis in both cell lines (Figure 3).

The Jab1 inhibitor CSN5i-3 leads to G1 phase arrest in human breast cancer cells. Based on our data, CSN5i-3 might be a potential therapeutic agent for breast cancer patients. We examined whether CSN5i-3 affects the cell cycle. PI staining was performed to detect the cell cycle distribution after CSN5i-3 treatment and showed that CSN5i-3 led to a notable G1 phase arrest in BT474 and SKBR3 cells (Figure 4).

The Jab1 inhibitor CSN5i-3 increases the expression of cleaved-PARP and p27 in human breast cancer cells. To understand the potential mechanism involved in the anticancer effect of CSN5i-3, Western blot was performed in BT474 and SKBR3 cells treated with $2 \mu \mathrm{M}$ CSN5i-3 for 24 hours. The western blot results showed that CSN5i-3 reduced the level of Jab1 and increased the levels of the apoptosis marker cleaved-PARP and the cell-cycle-related protein p27 in BT474 and SKBR3 cells (Figure 5). These data further proved the anticancer effect of CSN5i-3 in breast cancer cells.

CSN5i-3 oral administration inhibits BT474 xenograft growth in nude mice. To test the anticancer activity of CSN5i-3 in vivo, we subcutaneously injected the BT474 cells into the mammary fat pad area of the $\mathrm{BALB} / \mathrm{c}$ nude mice to 
A

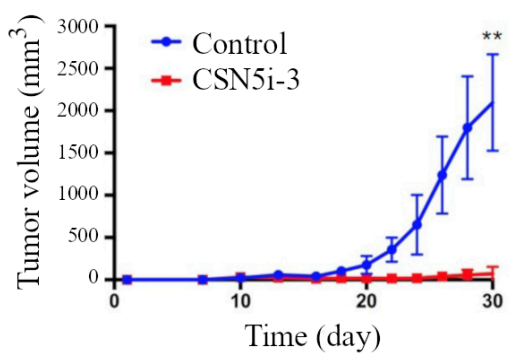

B

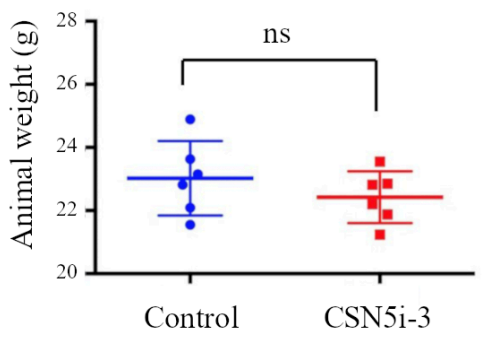

C

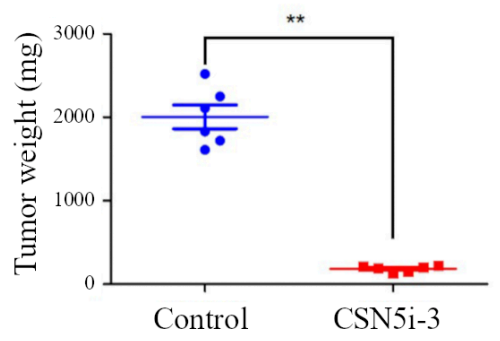

Figure 6. The tumor growth suppression effect of CSN5i-3 in human breast cancer cells in vivo. A) After treatment with CSN5I-3 for 21 days, we found that CSN5i-3 significantly suppressed the growth of BT474 tumors in mice $(\mathrm{p}<0.01)$. B) The weights of all the mice were measured, which showed that CSN5i-3 had limited effect on the weight of the mice $(23.23 \pm 0.61$ vs $22.54 \pm 0.38, p>0.05)$. C) After all mice were sacrificed, the tumor weights in both groups were measured. The mean tumor weights were $2007 \pm 141.9 \mathrm{mg}$ in the control group and $183.3 \pm 14.53 \mathrm{mg}$ in the treatment group (p<0.01).

establish a xenograft model. We showed that CSN5i-3 oral administration $(100 \mathrm{mg} / \mathrm{kg} / \mathrm{d})$ significantly inhibited the growth of the BT474 xenograft tumor (Figures 6A and 6C). Additionally, we recorded the weight of the mice, and found that CSN5i-3 treatment had a limited effect on the weight of the mice (Figure 6B). Taken together, our data demonstrated that CSN5i-3 treatment inhibited the growth of breast cancer tumors, and its toxicity was tolerable.

\section{Discussion}

Jab1 is considered a multifunctional protein that plays important roles in several vital biological processes, including DNA damage, cell proliferation, immune response and chemotherapy resistance [20]. In addition, Jab1 is frequently amplified/overexpressed in numerous cancer types, including gastric cancer, ovary cancer and breast cancer [17]. Previous researchers have reported that Jab1 knockdown suppressed cell proliferation and induced apoptosis in several malignant cells. Based on these important roles, it is logical to develop a specific drug targeting Jab1 in cancer cells [21]. CSN5i-3 is the first artificially synthesized Jab1-specific inhibitor and shows potent anticancer effects in several cancer cell lines [19]. However, the therapeutic effect of CSN5i-3 has not been studied in breast cancer.

Although the prognosis of breast cancer patients has been largely improved due to the development of clinical strategies and the emergence of novel therapeutic drugs, some patients, such as triple negative breast cancer patients, still demonstrate poor clinical outcomes. Promising therapeutic targets are still needed. In our study, we revealed the potent anticancer effect of the Jab1 inhibitor CSN5i-3 in human breast cancer cells in vitro and in vivo and showed that CSN5i-3 treatment significantly induced apoptosis and increased the level of apoptosis marker cleaved PARP. In addition, our data also revealed that CSN5i-3 treatment had limited effect on the weight of mice, which indicated that the CSN5i-3 toxicity was tolerable. This is important for the further evaluation of this drug. Further- more, we found that CSN5i-3 exposure induced G1 phase arrest, which might explain the anticancer effect of CSN5i-3 in breast cancer cells. Previous studies revealed that Jab1 can bind p27 and promote cell proliferation by leading to rapid nuclear export and subsequent degradation of p27 [22]. Our data also showed that CSN5i-3 treatment suppressed Jab1 expression and increased p21 expression, which might explain the G1 phase arrest caused by CSN5i-3 treatment.

In summary, we provide a series of data to prove that the Jab1 inhibitor CSN5i-3 might be a potent anticancer agent for breast cancer treatment. Further experiments are still needed to elucidate the potential underlying mechanisms by which CSN5i-3 inhibits proliferation and induces apoptosis in breast cancer cells.

Acknowledgements: This project was supported by a grant awarded to Hua Xiao by the Natural Science Foundation of Hunan Province (No. 2018JJ6108).

\section{References}

[1] SIEGEL RL, MILLER KD, JEMAL A. Cancer Statistics, 2017. CA Cancer J Clin 2017; 67: 7-30. https://doi.org/10.3322/ caac. 21387

[2] SENGUPTA R, HONEY K. AACR Cancer Progress Report 2018: Harnessing Research Discoveries for Patient Benefit. Clin Cancer Res 2018; 24: 4351. https://doi. org/10.1158/1078-0432.CCR-18-2756

[3] HARBECK N. Advances in targeting HER2-positive breast cancer. Curr Opin Obstet Gynecol 2018; 30: 55-59. https:// doi.org/10.1097/GCO.0000000000000431

[4] MENARD S, FORTIS S, CASTIGLIONI F, AGRESTI $\mathrm{R}$, BALSARI A. HER2 as a prognostic factor in breast cancer. Oncology 2001; 61 Suppl 2: 67-72. https://doi. org/10.1159/000055404

[5] PICCART-GEBHART MJ. Herceptin: the future in adjuvant breast cancer therapy. Anticancer Drugs 2001; 12 Suppl 4: S27-33. 
[6] SENDUR MA, AKSOY S, OZDEMIR NY, ZENGIN N, ALTUNDAG $\mathrm{K}$. What is the mechanism of progression with trastuzumab treatment--escape or resistance? Asian Pac J Cancer Prev 2012; 13: 5915-5916.

[7] ClARET FX, HiBI M, DHUT S, TODA T, KARIN M. A new group of conserved coactivators that increase the specificity of AP-1 transcription factors. Nature 1996; 383: 453457. https://doi.org/10.1038/383453a0

[8] KLEEMANN R, HAUSSER A, GEIGER G, MISCHKE R, BURGER-KENTISCHER A et al. Intracellular action of the cytokine MIF to modulate AP-1 activity and the cell cycle through Jab1. Nature 2000; 408: 211-216. https://doi. org/10.1038/35041591

[9] SHACKLEFORD TJ, CLARET FX. JAB1/CSN5: a new player in cell cycle control and cancer. Cell Div 2010; 5: 26. https://doi.org/10.1186/1747-1028-5-26

[10] BAE MK, AHN MY, JEONG JW, BAE MH, LEE YM et al. Jab1 interacts directly with HIF-1alpha and regulates its stability. J Biol Chem 2002; 277: 9-12. https://doi.org/10.1074/ jbc.C100442200

[11] WAN M, CAO X, WU Y, BAI S, WU L et al. Jab1 antagonizes TGF-beta signaling by inducing Smad4 degradation. EMBO Rep 2002; 3: 171-176. https://doi.org/10.1093/emboreports/kvf024

[12] OH W, LEE EW, SUNG YH, YANG MR, GHIM J et al. Jab1 induces the cytoplasmic localization and degradation of p53 in coordination with Hdm2. J Biol Chem 2006; 281: 1745717465. https://doi.org/10.1074/jbc.M601857200

[13] CHAMOVITZ DA, SEGAL D. JAB1/CSN5 and the COP9 signalosome. A complex situation. EMBO Rep 2001; 2: 96101. https://doi.org/10.1093/embo-reports/kve028
[14] AMBROGGIO XI, REES DC, DESHAIES RJ. JAMM: a metalloprotease-like zinc site in the proteasome and signalosome. PLoS Biol 2004; 2: E2. https://doi.org/10.1371/journal. pbio.0020002

[15] BIROL M, ECHALIER A. Structure and function of MPN (Mpr1/Pad1 N-terminal) domain-containing proteins. Curr Protein Pept Sci 2014; 15: 504-517. https://doi.org/10.2174/ 1389203715666140221095109

[16] GAO J, AKSOY BA, DOGRUSOZ U, DRESDNER G, GROSS B et al. Integrative analysis of complex cancer genomics and clinical profiles using the cBioPortal. Sci Signal 2013; 6: pl1. https://doi.org/10.1126/scisignal.2004088

[17] WANG L, ZHENG JN, PEI DS. The emerging roles of Jab1/CSN5 in cancer. Med Oncol 2016; 33: 90. https://doi. org/10.1007/s12032-016-0805-1

[18] PAN Y, YANG H, CLARET FX. Emerging roles of Jab1/ CSN5 in DNA damage response, DNA repair, and cancer. Cancer Biol Ther 2014; 15: 256-262. https://doi.org/10.4161/ cbt. 27823

[19] SCHLIERF A, ALTMANN E, QUANCARD J, JEFFERSON $\mathrm{AB}, \mathrm{ASSENBERG} \mathrm{R}$ et al. Targeted inhibition of the COP9 signalosome for treatment of cancer. Nat Commun 2016; 7: 13166. https://doi.org/10.1038/ncomms13166

[20] WEI N, SERINO G, DENG XW. The COP9 signalosome: more than a protease. Trends Biochem Sci 2008; 33: 592-600. https://doi.org/10.1016/j.tibs.2008.09.004

[21] WANG J, BARNES RO, WEST NR, OLSON M, CHU JE et al. Jab1 is a target of EGFR signaling in ERalpha-negative breast cancer. Breast Cancer Res 2008; 10: R51. https://doi. org/10.1186/bcr2105

[22] AHN J, HONG SA, LEE SE, KIM J, OH YS et al. Cytoplasmic localization of Jab1 and p27 Kip1 might be associated with invasiveness of papillary thyroid carcinoma. Endocr J 2009; 56: 707-713. https://doi.org/10.1507/endocrj.K08E-372 